\title{
Fossil fuel combustion, biomass burning and biogenic sources of fine carbonaceous aerosol in the Carpathian Basin
}

Imre Salma et al.

Correspondence to: Imre Salma (salma@chem.elte.hu)

The copyright of individual parts of the supplement might differ from the CC BY 4.0 License. 
Table S1. Mean apportionment multiplication factors of the coupled radiocarbon-levoglucosan marker model with SDs for regional background in the Carpathian Basin, suburban area and city centre of Budapest for different months of seasons.

\begin{tabular}{llllll}
\hline Factor & Site type & October & January & April & July \\
\hline$f_{1}$ & Region & $69 \pm 3$ & $75 \pm 3$ & $63 \pm 13$ & $74 \pm 9$ \\
$(\%)$ & Suburb & $68 \pm 6$ & $76 \pm 7$ & $50 \pm 6$ & $57 \pm 11$ \\
& Centre & $76 \pm 10$ & $74 \pm 2$ & $50 \pm 9$ & $58 \pm 7$ \\
$f_{2}$ & Region & $7.6 \pm 1.3$ & $14 \pm 1$ & $1.6 \pm 0.5$ & $0.41 \pm 0.15$ \\
$(\%)$ & Suburb & $8.3 \pm 1.7$ & $15 \pm 2$ & $3.5 \pm 1.1$ & $0.50 \pm 0.24$ \\
& Centre & $6.0 \pm 1.0$ & $13 \pm 1$ & $2.7 \pm 1.1$ & $0.61 \pm 0.18$ \\
$f_{3}$ & Region & $49 \pm 9$ & $93 \pm 6$ & $10 \pm 3$ & $2.4 \pm 1.0$ \\
$(\%)$ & Suburb & $53 \pm 12$ & $97 \pm 16$ & $21 \pm 7$ & $2.9 \pm 1.5$ \\
& Centre & $38 \pm 7$ & $90 \pm 8$ & $16 \pm 7$ & $3.6 \pm 1.1$ \\
$f_{4}$ & Region & $28 \pm 14$ & $5.3 \pm 2.4$ & $35 \pm 13$ & $24 \pm 14$ \\
$(\%)$ & Suburb & $14 \pm 11$ & $6.8 \pm 2.0$ & $36 \pm 8$ & $33 \pm 5$ \\
& Centre & $29 \pm 17$ & $19 \pm 4$ & $45 \pm 12$ & $31 \pm 6$ \\
\hline
\end{tabular}

Table S2. Median atmospheric concentrations of $\mathrm{SO}_{2}, \mathrm{NO}, \mathrm{NO}_{2}, \mathrm{CO}, \mathrm{O}_{3}$ and $\mathrm{PM}_{10}$ mass for regional background in the Carpathian Basin, suburban area and city centre of Budapest for the different months of seasons.

\begin{tabular}{llllll}
\hline Pollutant & Site type & October & January & April & July \\
\hline $\mathrm{SO}_{2}$ & Region & 0.44 & $\mathrm{NA}$ & $\mathrm{NA}$ & $\mathrm{NA}$ \\
$\left(\mu \mathrm{g} \mathrm{m}^{-3}\right)$ & Suburb & 2.2 & 2.0 & 1.35 & 1.50 \\
& Centre & 7.0 & 5.3 & 4.7 & 2.7 \\
$\mathrm{NO}$ & Region & $\mathrm{NA}$ & $\mathrm{NA}$ & $\mathrm{NA}$ & $\mathrm{NA}$ \\
$\left(\mu \mathrm{g} \mathrm{m}^{-3}\right)$ & Suburb & 8.4 & 4.7 & 1.40 & 1.05 \\
& Centre & 74 & 51 & 17.3 & 10.6 \\
$\mathrm{NO}_{2}$ & Region & 2.9 & $\mathrm{NA}$ & $\mathrm{NA}$ & $\mathrm{NA}$ \\
$\left(\mu \mathrm{g} \mathrm{m}^{-3}\right)$ & Suburb & 36 & 25 & 19.3 & 12.8 \\
& Centre & 52 & 37 & 57 & 31 \\
$\left.\mathrm{CO}_{(\mathrm{mg} \mathrm{m}}^{-3}\right)$ & Region & $\mathrm{NA}$ & $\mathrm{NA}$ & $\mathrm{NA}$ & $\mathrm{NA}$ \\
& Suburb & 0.50 & 0.60 & 0.78 & 0.69 \\
$\mathrm{O}_{3}$ & Centre & 0.69 & 0.56 & 0.61 & 0.33 \\
$\left(\mu \mathrm{g} \mathrm{m}^{-3}\right)$ & Region & NA & 37 & 103 & 92 \\
& Suburb & 12.4 & 15.5 & 67 & 74 \\
$\left.\mathrm{PM}_{10} \mathrm{mass}^{-3}\right)$ & Centre & 3.4 & 2.7 & 38 & 55 \\
$\left(\mu \mathrm{g} \mathrm{m}^{2}\right)$ & Region & 14.5 & $\mathrm{NA}$ & $\mathrm{NA}$ & $\mathrm{NA}$ \\
& Suburb & 30 & 29 & 39 & 21 \\
& Centre & 38 & 41 & 42 & 23 \\
\hline
\end{tabular}

NA - not available. 
Table S3. Mean atmospheric concentrations and $\mathrm{SDs}$ of $\mathrm{SO}_{2}, \mathrm{NO}, \mathrm{NO}_{2}, \mathrm{CO}, \mathrm{O}_{3}$ and $\mathrm{PM}_{10}$ mass for regional background in the Carpathian Basin, suburban area and city centre of Budapest for different months of seasons.

\begin{tabular}{llllll}
\hline Pollutant & Site type & October & January & April & July \\
\hline $\mathrm{SO}_{2}$ & Region & $0.67 \pm 0.38$ & $\mathrm{NA}$ & $\mathrm{NA}$ & $\mathrm{NA}$ \\
$\left(\mu \mathrm{g} \mathrm{m}^{-3}\right)$ & Suburb & $2.4 \pm 0.5$ & $2.1 \pm 0.7$ & $1.35 \pm 0.60$ & $1.55 \pm 0.35$ \\
& Centre & $7.0 \pm 0.2$ & $5.8 \pm 0.9$ & $4.7 \pm 0.8$ & $2.7 \pm 0.1$ \\
$\mathrm{NO}$ & Region & $\mathrm{NA}$ & $\mathrm{NA}$ & $\mathrm{NA}$ & $\mathrm{NA}$ \\
$\left(\mu \mathrm{g} \mathrm{m}^{-3}\right)$ & Suburb & $12.7 \pm 10.7$ & $11.1 \pm 10.1$ & $1.44 \pm 0.75$ & $1.08 \pm 0.46$ \\
& Centre & $75 \pm 16$ & $50 \pm 17$ & $17.6 \pm 3.2$ & $10.2 \pm 2.3$ \\
$\mathrm{NO}_{2}$ & Region & $3.1 \pm 1.4$ & $\mathrm{NA}$ & $\mathrm{NA}$ & $\mathrm{NA}$ \\
$\left(\mu \mathrm{g} \mathrm{m}^{-3}\right)$ & Suburb & $36 \pm 20$ & $31 \pm 12$ & $18.9 \pm 8.7$ & $14.9 \pm 7.3$ \\
& Centre & $50 \pm 8$ & $39 \pm 6$ & $52 \pm 11$ & $32 \pm 7$ \\
$\mathrm{CO}$ & Region & $\mathrm{NA}$ & $\mathrm{NA}$ & $\mathrm{NA}$ & $\mathrm{NA}$ \\
$\left(\mathrm{mg} \mathrm{m}^{-3}\right)$ & Suburb & $0.48 \pm 0.20$ & $0.66 \pm 0.18$ & $0.82 \pm 0.13$ & $0.61 \pm 0.17$ \\
& Centre & $0.68 \pm 0.09$ & $0.60 \pm 0.09$ & $0.61 \pm 0.07$ & $0.34 \pm 0.06$ \\
$\mathrm{O}_{3}$ & Region & $\mathrm{NA}$ & $41 \pm 21$ & $96 \pm 17$ & $90 \pm 11$ \\
$\left(\mu \mathrm{g} \mathrm{m}^{-3}\right)$ & Suburb & $21 \pm 18$ & $16.7 \pm 13.1$ & $67 \pm 12$ & $71 \pm 13$ \\
& Centre & $4.0 \pm 2.0$ & $3.5 \pm 2.1$ & $40 \pm 11$ & $56 \pm 9$ \\
$\mathrm{PM}_{10}$ mass & Region & $17.4 \pm 12.7$ & $\mathrm{NA}$ & $\mathrm{NA}$ & $\mathrm{NA}$ \\
$\left(\mu \mathrm{g} \mathrm{m}^{-3}\right)$ & Suburb & $36 \pm 25$ & $30 \pm 15$ & $35 \pm 11$ & $24 \pm 6$ \\
& Centre & $40 \pm 13$ & $40 \pm 12$ & $42 \pm 9$ & $27 \pm 7$ \\
\hline
\end{tabular}

NA - not available. 
Table S4. Mean air temperature ( $T$ ), relative humidity (RH), wind speed (WS) and daily maximum global solar radiation $\left(\mathrm{GRad}_{\max }\right)$ with SDs for regional background in the Carpathian Basin, suburban area and city centre of Budapest during the aerosol sampling campaign for different months of seasons.

\begin{tabular}{llllll}
\hline Property & Site type & October & January & April & July \\
\hline$T$ & Region & $8.4 \pm 1.9$ & $1.8 \pm 2.9$ & $17.1 \pm 2.6$ & $23 \pm 2$ \\
$\left({ }^{\circ} \mathrm{C}\right)$ & Suburb & $10.0 \pm 2.2$ & $2.5 \pm 3.3$ & $19.0 \pm 2.0$ & $24 \pm 2$ \\
& Centre & $12.9 \pm 1.9$ & $2.5 \pm 3.5$ & $19.9 \pm 1.0$ & $24 \pm 2$ \\
$\mathrm{RH}$ & Region & $88 \pm 10$ & $89 \pm 10$ & $64 \pm 8$ & $78 \pm 9$ \\
$(\%)$ & Suburb & $75 \pm 11$ & $79 \pm 8$ & $48 \pm 9$ & $56 \pm 8$ \\
& Centre & $79 \pm 4$ & $82 \pm 4$ & $49 \pm 9$ & $64 \pm 9$ \\
$\mathrm{WS}$ & Region & $1.61 \pm 1.14$ & $1.52 \pm 0.71$ & $1.45 \pm 0.57$ & $1.55 \pm 0.72$ \\
$\left(\mathrm{~m} \mathrm{~s}^{-1}\right)$ & Suburb & $1.64 \pm 1.10$ & $1.63 \pm 0.49$ & $1.27 \pm 0.23$ & $1.32 \pm 0.68$ \\
& Centre & $1.82 \pm 0.84$ & $2.1 \pm 0.4$ & $2.5 \pm 0.5$ & $3.9 \pm 1.7$ \\
$\mathrm{GRad}_{\max }$ & Region & NA & NA & NA & NA \\
$\left(\mathrm{kW} \mathrm{m}^{-2}\right)$ & Suburb & $0.31 \pm 0.15$ & $0.186 \pm 0.106$ & $0.77 \pm 0.09$ & $0.79 \pm 0.11$ \\
& Centre & NA & NA & $0.74 \pm 0.11$ & $0.76 \pm 0.15$ \\
\hline
\end{tabular}

NA - not available

The differences in the mean WS can be explained by non-equal heights of the meteorological sensors ( $3 \mathrm{~m}$ above the ground in the regional background and at the suburban area, and $12 \mathrm{~m}$ above the street level in the city centre). 
Table S5. Mean atmospheric concentrations and SDs of $\mathrm{PM}_{2.5}$ mass, elemental carbon (EC), organic carbon (OC), water-soluble organic carbon (WSOC), levoglucosan (LVG), mannosan (MAN), galactosan $(\mathrm{GAN})$, fraction of contemporary total carbon $\left(f_{\mathrm{c}}\right), \mathrm{K}, \mathrm{Ni}$ and $\mathrm{Pb}$ for regional background in the Carpathian Basin, suburban area and city centre of Budapest for different months of seasons.

\begin{tabular}{|c|c|c|c|c|c|}
\hline Constituent & Site type & October & January & April & July \\
\hline $\begin{array}{l}\mathrm{PM}_{2.5} \text { mass } \\
\left(\mu \mathrm{g} \mathrm{m}^{-3}\right)\end{array}$ & $\begin{array}{l}\text { Region } \\
\text { Suburb } \\
\text { Centre }\end{array}$ & $\begin{array}{l}16.3 \pm 8.3 \\
24 \pm 13 \\
29 \pm 11\end{array}$ & $\begin{array}{l}18.4 \pm 6.4 \\
25 \pm 9 \\
22 \pm 7\end{array}$ & $\begin{array}{l}9.7 \pm 2.7 \\
10.4 \pm 2.1 \\
13.5 \pm 3.1\end{array}$ & $\begin{array}{l}10.5 \pm 1.7 \\
11.6 \pm 2.8 \\
8.8 \pm 1.6\end{array}$ \\
\hline $\begin{array}{l}\mathrm{EC} \\
\left(\mu \mathrm{g} \mathrm{m}^{-3}\right)\end{array}$ & $\begin{array}{l}\text { Region } \\
\text { Suburb } \\
\text { Centre }\end{array}$ & $\begin{array}{l}0.41 \pm 0.24 \\
0.53 \pm 0.30 \\
1.08 \pm 0.37\end{array}$ & $\begin{array}{l}0.37 \pm 0.13 \\
0.73 \pm 0.26 \\
0.77 \pm 0.22\end{array}$ & $\begin{array}{l}0.21 \pm 0.06 \\
0.51 \pm 0.19 \\
0.84 \pm 0.22\end{array}$ & $\begin{array}{l}0.131 \pm 0.043 \\
0.41 \pm 0.16 \\
0.40 \pm 0.10\end{array}$ \\
\hline $\begin{array}{l}\mathrm{OC} \\
\left(\mu \mathrm{g} \mathrm{m}^{-3}\right)\end{array}$ & $\begin{array}{l}\text { Region } \\
\text { Suburb } \\
\text { Centre }\end{array}$ & $\begin{array}{l}3.0 \pm 1.8 \\
4.9 \pm 3.2 \\
6.4 \pm 1.9\end{array}$ & $\begin{array}{l}3.4 \pm 1.5 \\
5.4 \pm 2.4 \\
4.1 \pm 1.1\end{array}$ & $\begin{array}{l}2.1 \pm 0.5 \\
2.4 \pm 0.5 \\
2.8 \pm 0.8\end{array}$ & $\begin{array}{l}2.3 \pm 0.4 \\
2.7 \pm 0.4 \\
2.6 \pm 0.4\end{array}$ \\
\hline $\begin{array}{l}\text { WSOC } \\
\left(\mu \mathrm{g} \mathrm{m}^{-3}\right)\end{array}$ & $\begin{array}{l}\text { Region } \\
\text { Suburb } \\
\text { Centre }\end{array}$ & $\begin{array}{l}2.1 \pm 1.4 \\
2.6 \pm 1.7 \\
2.8 \pm 1.4\end{array}$ & $\begin{array}{l}2.1 \pm 1.0 \\
3.4 \pm 1.5 \\
2.6 \pm 0.7\end{array}$ & $\begin{array}{l}1.13 \pm 0.30 \\
1.27 \pm 0.34 \\
1.61 \pm 0.53\end{array}$ & $\begin{array}{l}1.62 \pm 0.29 \\
2.0 \pm 0.3 \\
2.0 \pm 0.4\end{array}$ \\
\hline $\begin{array}{l}\mathrm{LVG} \\
\left(\mu \mathrm{g} \mathrm{m}^{-3}\right)\end{array}$ & $\begin{array}{l}\text { Region } \\
\text { Suburb } \\
\text { Centre }\end{array}$ & $\begin{array}{l}0.189 \pm 0.134 \\
0.34 \pm 0.23 \\
0.38 \pm 0.06\end{array}$ & $\begin{array}{l}0.44 \pm 0.21 \\
0.74 \pm 0.44 \\
0.49 \pm 0.15\end{array}$ & $\begin{array}{l}0.0020 \pm 0.0013 \\
0.048 \pm 0.029 \\
0.049 \pm 0.024\end{array}$ & $\begin{array}{l}0.0081 \pm 0.0022 \\
0.0151 \pm 0.0100 \\
0.0113 \pm 0.0027\end{array}$ \\
\hline $\begin{array}{l}\text { MAN } \\
\left(\mathrm{ng} \mathrm{m}^{-3}\right)\end{array}$ & $\begin{array}{l}\text { Region } \\
\text { Suburb } \\
\text { Centre }\end{array}$ & $\begin{array}{l}19.2 \pm 5.7 \\
32 \pm 23 \\
29 \pm 8\end{array}$ & $\begin{array}{l}24 \pm 14 \\
46 \pm 25 \\
23 \pm 7\end{array}$ & $\begin{array}{l}2.6 \pm 0.2 \\
3.4 \pm 1.7 \\
5.5 \pm 2.6\end{array}$ & $\begin{array}{l}<1.2 \\
<1.2 \\
<1.2\end{array}$ \\
\hline $\begin{array}{l}\text { GAN } \\
\left(\mathrm{ng} \mathrm{m}^{-3}\right)\end{array}$ & $\begin{array}{l}\text { Region } \\
\text { Suburb } \\
\text { Centre }\end{array}$ & $\begin{array}{l}\text { NA } \\
14.1 \pm 10.1 \\
12.3 \pm 2.7\end{array}$ & $\begin{array}{l}11.9 \pm 6.0 \\
22 \pm 11 \\
14.1 \pm 3.9\end{array}$ & $\begin{array}{l}1.27 \pm 0.25 \\
1.47 \pm 0.84 \\
1.32 \pm 0.35\end{array}$ & $\begin{array}{l}<0.5 \\
<0.5 \\
<0.5\end{array}$ \\
\hline $\begin{array}{l}f_{\mathrm{c}} \\
(\%)\end{array}$ & $\begin{array}{l}\text { Region } \\
\text { Suburb } \\
\text { Centre }\end{array}$ & $\begin{array}{l}69 \pm 3 \\
68 \pm 6 \\
76 \pm 6\end{array}$ & $\begin{array}{l}74 \pm 3 \\
76 \pm 7 \\
74 \pm 2\end{array}$ & $\begin{array}{l}63 \pm 13 \\
50 \pm 6 \\
50 \pm 9\end{array}$ & $\begin{array}{l}74 \pm 9 \\
57 \pm 11 \\
59 \pm 7\end{array}$ \\
\hline $\begin{array}{l}\mathrm{K} \\
\left(\mu \mathrm{g} \mathrm{m}^{-3}\right)\end{array}$ & $\begin{array}{l}\text { Region } \\
\text { Suburb } \\
\text { Centre }\end{array}$ & $\begin{array}{l}0.188 \pm 0.071 \\
0.22 \pm 0.11 \\
0.27 \pm 0.07\end{array}$ & $\begin{array}{l}0.26 \pm 0.09 \\
0.28 \pm 0.14 \\
0.28 \pm 0.05\end{array}$ & $\begin{array}{l}0.107 \pm 0.043 \\
0.105 \pm 0.031 \\
0.112 \pm 0.031\end{array}$ & $\begin{array}{l}0.081 \pm 0.003 \\
0.075 \pm 0.016 \\
0.057 \pm 0.013\end{array}$ \\
\hline $\begin{array}{l}\mathrm{Ni} \\
\left(\mathrm{ng} \mathrm{m}^{-3}\right)\end{array}$ & $\begin{array}{l}\text { Region } \\
\text { Suburb } \\
\text { Centre }\end{array}$ & $\begin{array}{l}1.01 \pm 0.63 \\
0.97 \pm 0.30 \\
1.25 \pm 0.36\end{array}$ & $\begin{array}{l}0.71 \pm 0.13 \\
0.98 \pm 0.59 \\
0.86 \pm 0.47\end{array}$ & $\begin{array}{l}1.31 \pm 0.32 \\
1.26 \pm 0.21 \\
1.60 \pm 0.23\end{array}$ & $\begin{array}{l}1.23 \pm 0.48 \\
1.35 \pm 0.54 \\
1.08 \pm 0.13\end{array}$ \\
\hline $\begin{array}{l}\mathrm{Pb} \\
\left(\mathrm{ng} \mathrm{m}^{-3}\right)\end{array}$ & $\begin{array}{l}\text { Region } \\
\text { Suburb } \\
\text { Centre }\end{array}$ & $\begin{array}{l}3.9 \pm 0.9 \\
6.4 \pm 3.0 \\
6.7 \pm 1.6\end{array}$ & $\begin{array}{l}4.1 \pm 2.4 \\
7.7 \pm 3.9 \\
5.5 \pm 2.5\end{array}$ & $\begin{array}{l}3.5 \pm 1.9 \\
4.8 \pm 1.8 \\
5.2 \pm 1.9\end{array}$ & $\begin{array}{l}3.9 \pm 3.0 \\
4.5 \pm 2.9 \\
2.4 \pm 1.5\end{array}$ \\
\hline
\end{tabular}

NA - not available. 
Table S6. Coefficients of correlation between $\mathrm{EC}_{\mathrm{FF}}, \mathrm{OC}_{\mathrm{FF}}, \mathrm{EC}_{\mathrm{BB}}, \mathrm{OC}_{\mathrm{BB}}$ and $\mathrm{OC}_{\mathrm{BIO}}$ on the one side and $\mathrm{K}, \mathrm{NO}$, and $T$ on the other side for regional background in the Carpathian Basin, suburban area and city centre of Budapest.

\begin{tabular}{lllllll}
\hline $\begin{array}{l}\text { Variable } \\
\text { (unit) }\end{array}$ & Site type & $\begin{array}{l}\mathrm{EC}_{\mathrm{FF}} \\
\left(\mu \mathrm{g} \mathrm{m}^{-3}\right)\end{array}$ & $\begin{array}{l}\mathrm{OC}_{\mathrm{FF}} \\
\left(\mu \mathrm{g} \mathrm{m}^{-3}\right)\end{array}$ & $\begin{array}{l}\mathrm{EC}_{\mathrm{BB}} \\
\left(\mu \mathrm{g} \mathrm{m}^{-3}\right)\end{array}$ & $\begin{array}{l}\mathrm{OC}_{\mathrm{BB}} \\
\left(\mu \mathrm{g} \mathrm{m}^{-3}\right)\end{array}$ & $\begin{array}{l}\mathrm{OC}_{\mathrm{BIO}} \\
\left(\mu \mathrm{g} \mathrm{m}^{-3}\right)\end{array}$ \\
\hline $\mathrm{K}$ & Region & 0.02 & 0.73 & 0.95 & 0.95 & -0.26 \\
$\left(\mu \mathrm{g} \mathrm{m}^{-3}\right)$ & Suburb & -0.69 & 0.51 & 0.90 & 0.91 & 0.20 \\
& Centre & -0.22 & 0.44 & 0.86 & 0.86 & 0.20 \\
$\mathrm{NO}$ & Region & $\mathrm{NA}$ & $\mathrm{NA}$ & $\mathrm{NA}$ & $\mathrm{NA}$ & $\mathrm{NA}$ \\
$\left(\mu \mathrm{g} \mathrm{m}^{-3}\right)$ & Suburb & -0.36 & 0.93 & 0.60 & 0.60 & 0.42 \\
& Centre & -0.17 & 0.39 & 0.76 & 0.77 & 0.44 \\
$T$ & Region & -0.37 & -0.58 & -0.87 & -0.85 & 0.60 \\
$(\mathrm{~K})$ & Suburb & 0.59 & -0.24 & -0.85 & -0.87 & 0.24 \\
& Centre & 0.53 & -0.08 & -0.79 & -0.80 & 0.34 \\
\hline
\end{tabular}

Table S7. Mean contributions with SDs of fossil fuel (FF) combustion, biomass burning (BB), biogenic sources (Bio) and unaccounted part (UnA) to the $\mathrm{PM}_{2.5}$ mass in \% as rough estimates for regional background in the Carpathian Basin, suburban area and city centre of Budapest for different months of seasons.

\begin{tabular}{llllll}
\hline Environment & Source & October & January & April & July \\
\hline Regional & FF & $12 \pm 3$ & $8 \pm 2$ & $13 \pm 6$ & $8 \pm 3$ \\
background & BB & $14 \pm 3$ & $21 \pm 3$ & $3 \pm 1$ & $<2$ \\
& Bio & $14 \pm 5$ & $<2$ & $23 \pm 5$ & $24 \pm 4$ \\
& UnA & $60 \pm 8$ & $70 \pm 4$ & $61 \pm 8$ & $67 \pm 3$ \\
Suburban & FF & $14 \pm 5$ & $8 \pm 3$ & $20 \pm 5$ & $15 \pm 4$ \\
area & BB & $16 \pm 6$ & $23 \pm 3$ & $5 \pm 3$ & $<2$ \\
& Bio & $13 \pm 5$ & $3 \pm 2$ & $16 \pm 4$ & $24 \pm 3$ \\
& UnA & $58 \pm 13$ & $67 \pm 4$ & $59 \pm 9$ & $61 \pm 5$ \\
City & FF & $9 \pm 5$ & $9 \pm 2$ & $18 \pm 6$ & $20 \pm 4$ \\
centre & BB & $11 \pm 2$ & $22 \pm 4$ & $4 \pm 2$ & $<2$ \\
& Bio & $16 \pm 3$ & $3 \pm 1$ & $18 \pm 6$ & $31 \pm 6$ \\
& UnA & $63 \pm 3$ & $67 \pm 4$ & $60 \pm 8$ & $48 \pm 6$ \\
\hline
\end{tabular}

